\title{
Stability of a short Rayleigh length laser resonator
}

\author{
P. P. Crooker, ${ }^{*}$ J. Blau, and W. B. Colson \\ Physics Department, Naval Postgraduate School, 833 Dyer Road, Monterey, California 93943, USA
}

(Received 4 January 2005; published 22 April 2005)

\begin{abstract}
Motivated by the prospect of constructing a short Rayleigh length free-electron laser in a high-vibration environment, we demonstrate the use of a collection of rays to study the effect of mirror vibration and distortion on the behavior of the fundamental optical mode of a cold-cavity resonator. We find that the ray collection accurately describes both on-axis and off-axis optical beams. We show that a tilt or transverse shift of a mirror causes the optical mode to rock about the original resonator axis, while a longitudinal mirror shift or a change in the mirror's radius of curvature causes the beam diameter at a mirror to successively dilate and contract on the mirror. Results are in excellent agreement with analytic calculations and wave front propagation simulations as long as the mirrors remain large with respect to the beam diameter.
\end{abstract}

DOI: $10.1103 /$ PhysRevSTAB.8.040703

PACS numbers: 61.30.Cz, 64.70.Md

\section{INTRODUCTION}

Some proposed designs for a high-power free-electron laser (FEL) call for a short Rayleigh length optical resonator in order to reduce the system size while minimizing heat damage to the mirrors [1-3]. These designs raise concerns about mode stability, in particular, the sensitivity to motions of the mirrors. This paper presents a study of the effect on beam behavior of mirror motion and mirror radius change, particularly as they affect short Rayleigh length resonators.

We utilize a collection of propagating rays to study the beam behavior in the resonator. The method is applicable when the cavity mirrors are unperturbed and the beam corresponds to an eigenmode of the resonator. However, the method is easily adaptable to situations where the beam is not a resonator eigenmode, and we show how the beam evolves for several cavity distortions: mirror tilt, transverse and longitudinal shifts in mirror position, and changes in mirror focal length. In order to isolate resonator effects alone, our results are for a resonator with no gain. Since mirror motions are relatively slow $(\sim \mathrm{ms})$ compared with the optical round trip time $(\sim \mathrm{ns})$, the motions are assumed to be fixed over many passes of the beam through the resonator.

In general, the optical beam in a laser resonator retraces itself - it is an eigenmode of the resonator. If a mirror is misaligned or distorted, however, the resonator eigenmode will be redefined and the existing optical beam will tend to walk around the mirrors [4]. For sufficiently large misalignment, the beam radius may increase indefinitelyi.e., the resonator may become unstable. These effects are most pronounced for short Rayleigh length resonators, which are already near the stability limit. In practical terms, mirror misalignment and distortion may cause the beam displacement to exceed the size of the mirrors,

*Email address: ppcrooke@nps.edu thereby creating beam loss and distortion of the beam envelope.

\section{SIMULATION TECHNIQUES}

We assume a resonator with two identical mirrors of focal length $F$ separated by distance $S$ (Fig. 1) initially aligned along the $z$ axis. The resonator axis is defined as the line connecting the two centers of curvature of the mirrors and the fundamental Gaussian optical eigenmode is characterized by Rayleigh length $Z_{0}=S(F / S-1 / 4)^{1 / 2}$. Let the resonator initially contain such an eigenmode with wavelength $\lambda$. We adopt the convention where all longitudinal lengths are normalized by cavity length $S$; all transverse lengths are normalized by $(\lambda S / \pi)^{1 / 2}$; angles are normalized by $(\lambda / \pi S)^{1 / 2}$; and time $t$ is normalized by $S / c$, the time for a one-way pass through the resonator. Using lower case symbols for normalized lengths, $z_{0}=$ $(f-1 / 4)^{1 / 2}$ and the transverse behavior of the electric field amplitude is given by [5]

$$
E(x, y)=A e^{-\left(x^{2}+y^{2}\right) / w^{2}},
$$

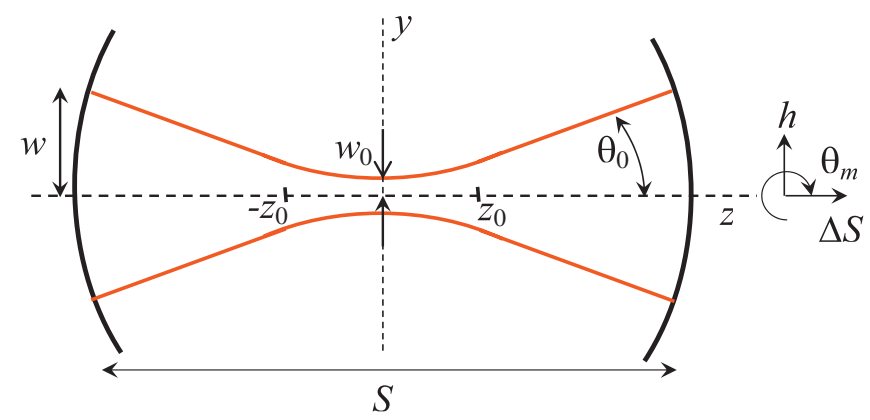

FIG. 1. (Color) Resonator with Gaussian mode characterized by Rayleigh length $z_{0}$. Vibrations of the right-hand mirror include tilt $\theta_{m}$, transverse shift $h$, longitudinal shift $\Delta S$, and focal length change $\Delta f$ (not shown). 
where the transverse $1 / e$ radius of the beam at any $z$ is

$$
w=\left(z_{0}+z^{2} / z_{0}\right)^{1 / 2} .
$$

For a typical resonator with $S=10 \mathrm{~m}$ and $\lambda=1 \mu \mathrm{m}$, the transverse scaling length is $1.8 \mathrm{~mm}$, the scaling angle is $0.18 \mathrm{mrad}$, and the scaling time is $33 \mathrm{~ns}$.

Note that any tilt or transverse shift of a mirror redefines the cavity axis, so that the initial optical beam becomes off axis: it is no longer an eigenmode and on successive passes will not retrace itself. Also note that any change in a mirror's focal length or longitudinal position shifts the resonator's natural $z_{0}$ away from that of the beam. Again, the beam is no longer a resonator eigenmode, and it will change its shape with each pass through the resonator.

Simulation of an optical beam can be done several ways, as we show next.

\section{A. Ray-tracing technique}

We represent the Gaussian beam by a random collection of rays Gaussian distributed in both transverse position $y$ and angle $\theta$. If the distribution functions for $y$ and $\theta$ are chosen correctly, the ray collection will mimic the amplitude behavior of a true Gaussian beam [6]. Let these distributions be combined into a joint probability density $f(y, \theta)$. At the beam waist, $y$ and $\theta$ are uncorrelated:

$$
f(y, \theta)=\frac{1}{\pi y_{0} \theta_{0}} e^{-\left(y^{2} / y_{0}^{2}+\theta^{2} / \theta_{0}^{2}\right)}
$$

The expressions for $y_{0}$ and $\theta_{0}$ are found by comparing (3) to the expression for the transverse behavior of the electric field amplitude given by (1). From (2), the width of the Gaussian beam at its waist $(z=0)$ is just $y_{0}=w_{0}=z_{0}^{1 / 2}$. The angular spread $\theta_{0}$ can be seen to be the beam opening angle $w / z$ for $z \gg z_{0}$, as shown in Fig. 1. From (2) we get $\theta_{0}=1 / z_{0}^{1 / 2}$. The joint probability for the beam amplitude becomes, therefore,

$$
f(y, \theta)=\frac{1}{\pi} e^{-\left(y^{2}+z_{0}^{2} \theta^{2}\right) / z_{0}} .
$$

It is interesting to note that, if we square $f(y, \theta)$ so that it represents a photon probability density, the resulting normalized standard deviations $\sigma_{y}=\left(z_{0} / 4\right)^{1 / 2}$ in $y$ and $\sigma_{\theta}=$ $\left(1 / 4 z_{0}\right)^{1 / 2}$ in $\theta$ exactly satisfy the uncertainty relation $\sigma_{y} \sigma_{\theta}=1 / 4$ as they must [6].

To simulate the propagation and reflection of the beam, $y$ and $\theta$ become components of a $2 \times 1$ column vector and are propagated numerically using $2 \times 2 A B C D$ ray matrices [5]. For our purposes, however, the usual matrices for drift, reflection, etc., must also incorporate the effect of mirror tilt, mirror shift, and focal length change. In the limit of a large number of rays, the transverse density of the propagated rays becomes proportional to the actual amplitude behavior of a Gaussian beam. The method is simple to program and completely adaptable to resonators with small $z_{0}$. In addition, the method can handle situations where reorientation or refocusing of a mirror produces beams which are not resonator eigenmodes.

\section{B. Wave propagation technique}

The wave propagation technique [1] provides a useful comparison with the ray technique. Here the complex amplitude $a(x, y, z)$ of a Gaussian beam is set up on a computational grid at the beam waist and then propagated numerically by the paraxial wave equation $\partial_{z} a=$ $(-i / 4) \nabla_{\perp}^{2} a$. Mirrors can be handled by a suitable phase shift quadratic in mirror radius at the mirror position. This method includes diffractive effects and, if coupling to the electrons is included, can describe laser gain and finite mirror size. It can also accommodate tilted, shifted, and distorted mirrors. The method is, however, computationally intensive, especially in a short Rayleigh length resonator where the grid must be small and fine at the beam waist and large and coarse at the mirrors. To overcome this limitation, the simulation uses a coordinate system that expands with the natural diffraction of the Gaussian mode [7].

\section{Gaussian beam theory}

Gaussian beam theory [5] provides another check on the ray technique. A Gaussian beam may be described analytically by its complex beam radius $q=z+i z_{0}$, where $z$ is the distance from the beam waist. Since $1 / q=1 / R-$ $i / w^{2}$, one can immediately obtain the transverse beam radius $w$ and the wave front curvature radius $R$. Propagation or focusing changes $q$ into $q^{\prime}$; this is accomplished using the $A B C D$ matrix elements in the form $q^{\prime}=$ $(A q+B) /(C q+D)$. This method will accommodate longitudinal mirror shift and focal length change since it only works along the beam axis. However, if a new beam axis is determined by ray tracing, it can also describe the effects of tilt and transverse shift of the mirrors.

\section{MIRROR TILT AND SHIFT}

We now let the right-hand mirror (focal length $f=z_{0}^{2}+$ $1 / 4)$ undergo tilt $\theta_{m}$ and/or transverse shift $h$ and investigate the subsequent behavior of the Gaussian beam. The immediate effect is that the reflection angle of any ray incident on the mirror will be increased by $2 \theta_{m}+h / f$. In the paraxial limit with infinite mirror width, the resonator will remain stable, but a new resonator axis will be defined which tilts with respect to the old axis by amount $\phi$, where

$$
\phi=-\left[\left(1+4 z_{0}^{2}\right) \theta_{m}+2 h\right] /\left(8 z_{0}^{2}\right) .
$$

The optical beam, which initially was an eigenmode of the old resonator, will now be tilted with respect to the new axis and is no longer an eigenmode of the realigned reso- 
nator. Consequently, over many reflections, the beam angle changes in a rocking fashion, depending on the value of $z_{0}$.

The effect of the rocking over many passes is to make the beam position walk up and down the mirror. If $y_{n}$ is the position of the beam center on the right mirror after $n$ round trips [5],

$$
y_{n}=C_{1}[1-\cos (\alpha n)]+C_{2} \sin (\alpha n)
$$

where

$$
\begin{gathered}
\alpha=\cos ^{-1}\left(\frac{2 f^{2}-4 f+1}{2 f^{2}}\right), \\
C_{1}=\left(\frac{2 f-1}{4 f-1}\right)\left(h+2 f \theta_{m}\right), \\
C_{2}=\frac{-\left(h+2 f \theta_{m}\right)}{\sqrt{4 f-1}},
\end{gathered}
$$

and where $f=z_{0}^{2}+1 / 4$.

Figure 2 shows the result of a ray-tracing simulation involving mirror tilt. The beam is started on axis with the right mirror tilted. Successive reflections of the beam are unfolded, so that the horizontal axis is normalized time $\tau=c t / S$. The beam angle changes with each reflection, depending on $z_{0}$ and, for this figure, $\theta_{m}$. From (6), the beam center has a maximum deflection $y_{c}$ which is proportional to $\theta_{m}$ and $h$ :

$$
y_{c}=\frac{\left(4 z_{0}^{2}+1\right) \theta_{m}+2 h}{8 z_{0}^{2}} .
$$

For the small $z_{0}$ case, with which we are concerned here, $y_{c}$ is a strong function of $z_{0}$. We show this dependence in

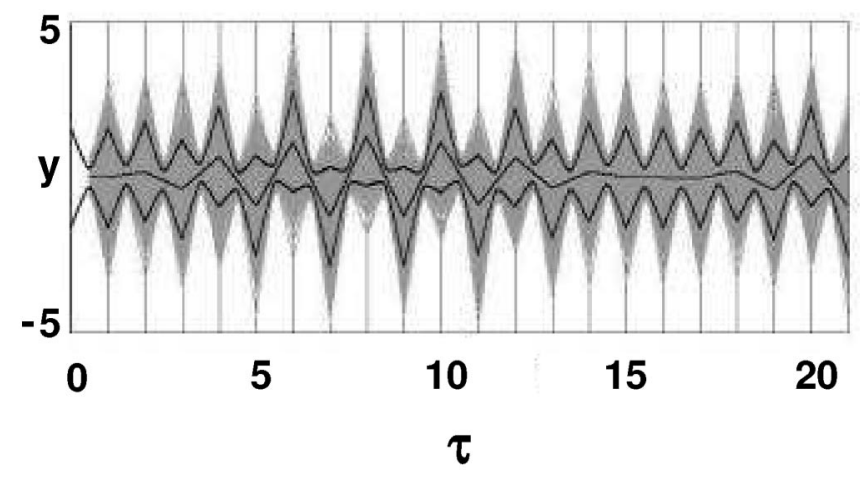

FIG. 2. Evolution of an optical beam in a resonator with $z_{0}=$ $0.1, \theta_{m}=0.05$, and $h=0$. The $y$ axis is the normalized transverse distance, and $\tau$ is the normalized time. Each vertical line corresponds to a mirror, with successive reflections unfolded to see the overall behavior. The shaded area shows the trajectories of 1000 random rays; the center line is the center of the optical beam; and the top and bottom lines, calculated from beam theory, correspond to the radius $w$ for the Gaussian mode. The effect of mirror tilt is to make the beam rock back and forth on the resonator mirrors.

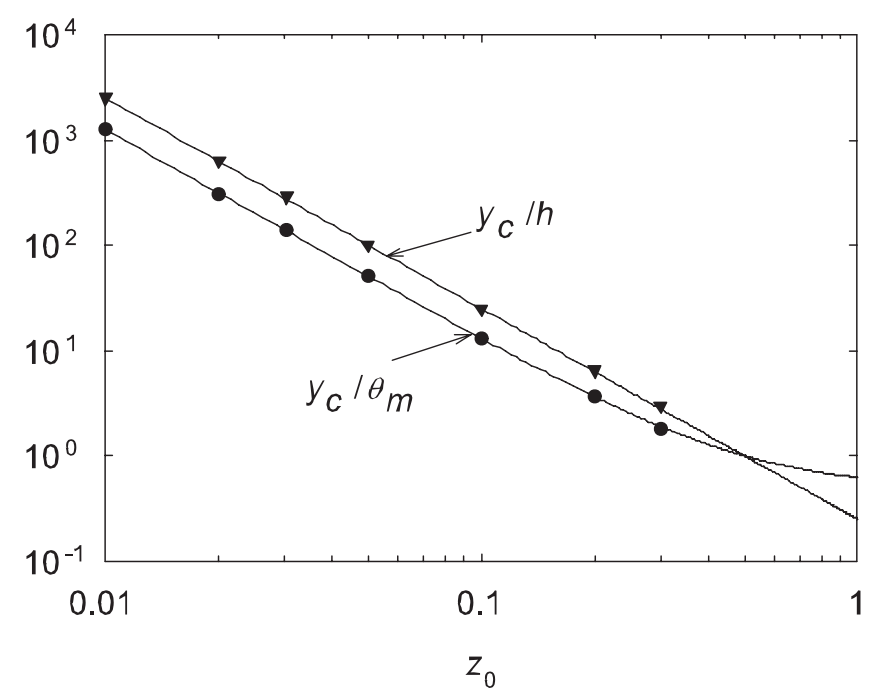

FIG. 3. Dependence on Rayleigh length $z_{0}$ of the maximum excursion $y_{c}$ of the beam center from the original cavity axis when a mirror tilts by $\theta_{m}$ or undergoes transverse shift $h$. Tilt and shift are plotted separately. The agreement between beam theory (lines) and wave simulations (points) is excellent over many orders of magnitude. For an FEL with $S=10 \mathrm{~m}$ and $\lambda=1 \mu \mathrm{m}$, $y_{c}=10$ corresponds to $1.8 \mathrm{~cm}$.

Fig. 3 where $y_{c} / \theta_{m}$ and $y_{c} / h$ are plotted separately against $z_{0}$. Clearly the ray theory (lines) is in excellent agreement with wave theory (data points) over many orders of magnitude; in fact, we have observed good agreement down to $z_{0}=0.0001$. In an actual FEL, as $z_{0}$ becomes smaller the transverse excursions will become comparable to a typical mirror radius and the beam will walk off the mirrors.

\section{LONGITUDINAL MIRROR SHIFT}

Let the resonator contain a Gaussian beam which is a resonator eigenmode with Rayleigh length $z_{0}$. Since $z_{0}$ is small, the mirror focal lengths $f=z_{0}^{2}+1 / 4$ are already only slightly larger than the resonator stability $\operatorname{limit} f_{\min }=$ $1 / 4$. Let the right mirror shift by fractional amount $\Delta s=$ $\Delta S / S$ in the $z$ direction. Successive reflections of the beam remain on axis, but the Rayleigh lengths of the beam and the resonator eigenmode will no longer be equal. If $\Delta s$ is positive (cavity length increases), the normalization length becomes $S+\Delta S$, and the normalized focal lengths decrease to $f^{\prime}=f /(1+\Delta s)$. If $f^{\prime}<1 / 4$, the resonator becomes unstable and the beam will expand without limit. The maximum allowable value for $\Delta s$ is therefore $\Delta s_{\max }=$ $4 f-1=4 z_{0}^{2}$.

If $\Delta s<\Delta s_{\max }$, the resonator remains stable but the beam no longer retraces itself with succeeding passes, as shown in Fig. 4. With each pass, the beam radius at the mirrors expands or contracts, with maximum beam radius $y_{\max }$ depending on both $\Delta s$ and $z_{0}$. Figure 5 shows the effect of varying $\Delta s$ for several $z_{0}$. For $\Delta s<\Delta s_{\max }$, the effect on $y_{\max }$ is small. However, as $\Delta s$ approaches $\Delta s_{\max }$, $y_{\max }$ increases and finally diverges at $\Delta s_{\max }$. 


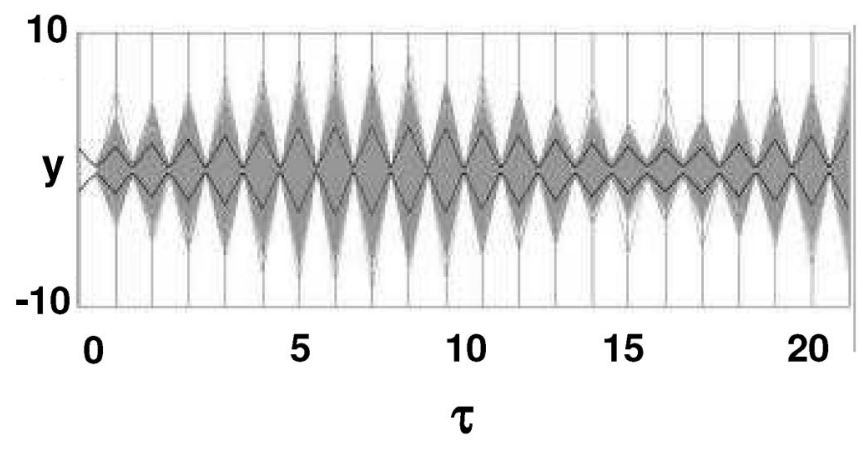

FIG. 4. Evolution of an optical beam in a resonator with $z_{0}=$ 0.1 and right mirror shift $\Delta s=0.031$. The axes are the same as Fig. 2. The gray areas are the trajectories of 1000 random rays; the dotted lines, calculated from beam theory, correspond to the radius $w$ of the Gaussian mode. The beam remains on axis, but expands and contracts with successive reflections.

For $\Delta s<0$ (resonator length decreases), the resonator remains stable but the beam width again expands dramatically as the difference between the Rayleigh lengths of the beam and the resonator eigenmode becomes large.

\section{MIRROR DISTORTION}

Now let the focal length $f$ of the right-hand mirror in the previously undistorted resonator change by amount $\Delta f / f$. Focal length change could be caused, for example, by heating from a very intense optical beam or vibrations of the mirror substrate. Since the mirror focal lengths are unequal, the mode waist of the resonator eigenmode will move away from the resonator center. The effect is to change the resonator eigenmode so that it no longer cor-

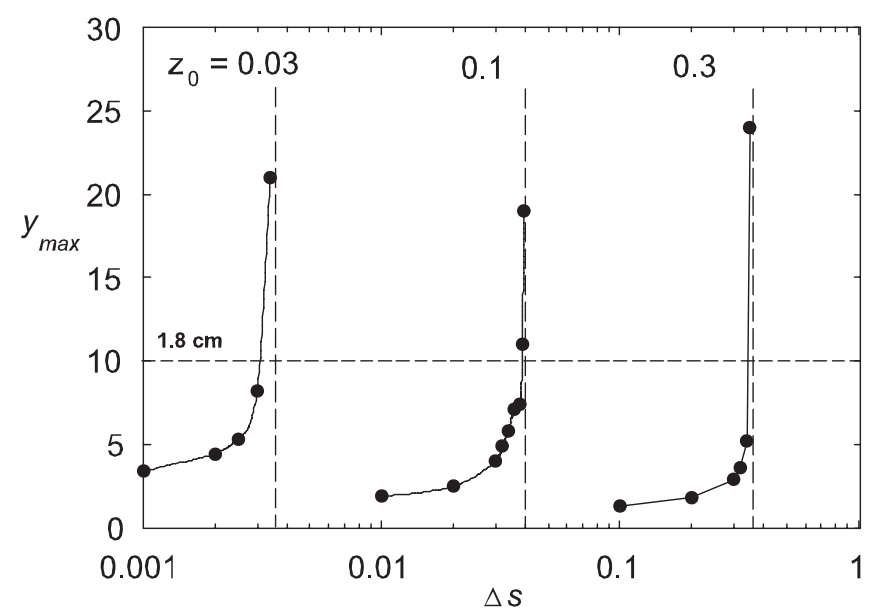

FIG. 5. Maximum beam radius $y_{\max }$ for fractional mirror shift $\Delta s$ of the right-hand mirror at several values of $z_{0}$. As $\Delta s$ increases, $y_{\max }$ diverges where the cavity becomes spherical at $\Delta s_{\max }=4 z_{0}^{2}$ (vertical dashed lines). The data points are taken from ray and beam simulations; the solid lines are guides to the eye. For an FEL with $S=10 \mathrm{~m}$ and $\lambda=1 \mu \mathrm{m}, y_{\max }=10$ corresponds to $1.8 \mathrm{~cm}$.

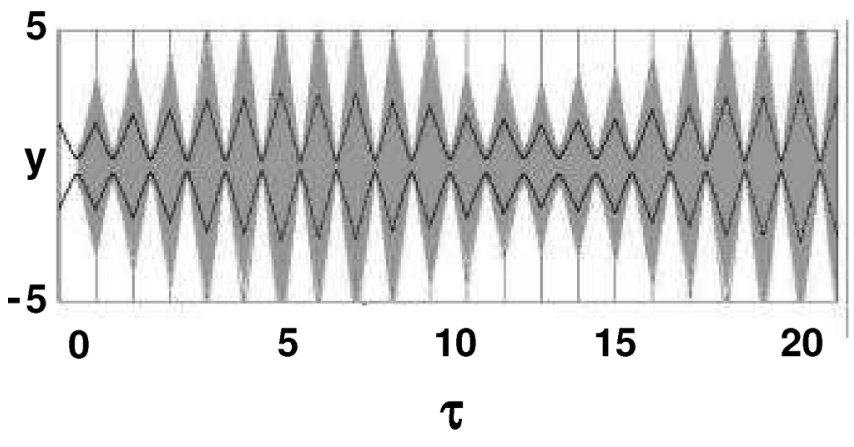

FIG. 6. Evolution of an optical beam in a resonator as a function of normalized time $\tau$, with $z_{0}=0.1$ and right mirror fractional focal length change $\Delta f / f=-0.05$. The axes and lines are the same as Fig. 2. As in Fig. 4, the beam expands and contracts with successive reflections.

responds to that of the original beam. Consequently, the beam radius on the mirror will expand and contract with each subsequent reflection, as shown in Fig. 6. In addition, if $\Delta f / f$ is negative (a decrease in the mirror focal length) and made too large, the resonator will no longer be stable and the beam will diverge indefinitely. The stability criterion is $\Delta f / f>-8 z_{0}^{2} /\left(1+4 z_{0}^{2}\right)$.

Figure 7 shows the results from our simulations. The beam radius at the mirror is $y_{\max }$, as before. As $\Delta f / f$ is made increasingly negative, $y_{\max }$ increases slowly as the threshold for resonator instability (vertical dashed lines) is approached, and then diverges sharply where the cavity becomes spherical and unstable.

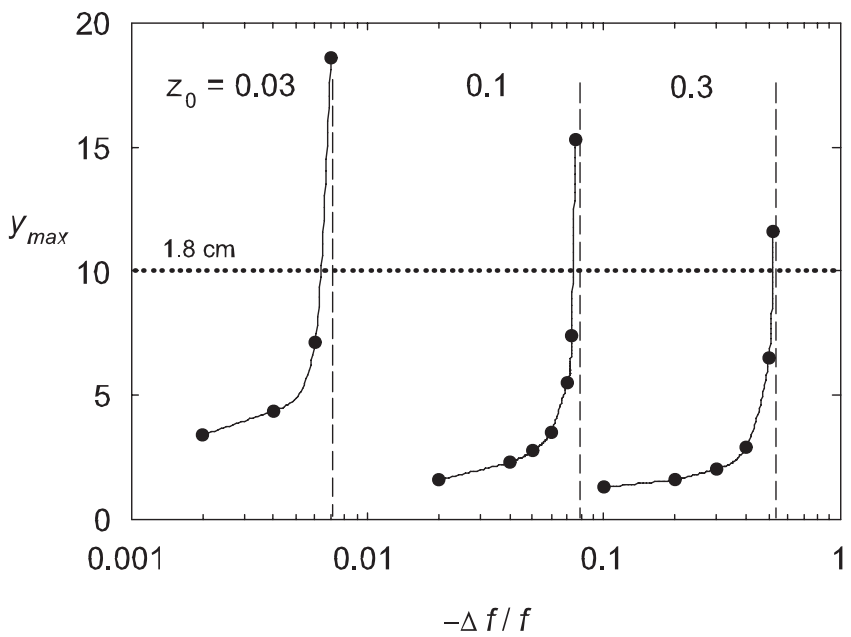

FIG. 7. Maximum beam radius $y_{\max }$ for fractional focal length change $\Delta f / f$ of the right-hand mirror at several values of $z_{0}$. The minus sign in front of $\Delta f / f$ indicates the focal length is decreasing. As the focal length decreases, $y_{\max }$ diverges where the cavity becomes spherical at $\Delta f / f=-8 z_{0}^{2} /\left(1+4 z_{0}^{2}\right)$ (vertical dashed lines). The points are taken from ray simulations and beam calculations; the solid lines are guides to the eye. 


\section{EFFECT OF FINITE MIRROR RADIUS}

It is tempting to apply the ray simulation technique to situations where the mirror's transverse radius is finite [8-13]. If the beam power is proportional to the number of rays, one can observe a decrease in power as those rays which fall outside the mirror radius are excluded. Figure 8 shows the result of limiting the radii of both mirrors. We take $z_{0}=0.1$ so that, from (2), the beam radius at the mirror is $w=1.6$, and we set the transverse mirror radius $r_{m}=2$. Mirror tilt and displacement are both zero. Rays falling outside the mirror are eliminated successively on each pass and the power in the beam is monotonically decreased. After several passes, however, the remaining rays lie entirely on the mirrors and the power remains constant and nonzero.

A wave simulation under the same conditions comes to a different conclusion (Fig. 8). Here the beam power continues to decrease exponentially as the outer portions of the wave fronts, which lie outside the mirror radius, are lost on each pass. The explanation for the difference in the ray and wave methods is that the elimination of rays in the $z \gg z_{0}$ region artificially reduces the beam emittance $\Delta y \Delta \theta$, which was already at the limit of the uncertainty principle, $\Delta y \Delta \theta=1$ in our normalized notation. Narrowing the beam to accommodate the finite mirror at $z \gg z_{0}$ effectively reduces the angular spread $\Delta \theta$ (by throwing out the high-angle rays) with little change in $\Delta y$. In reality, there must be a corresponding increase in $\Delta y$, caused by diffraction, to satisfy the uncertainty principle. The wave method includes this diffraction, but the ray treatment, which has no diffractive effects, does not.

Agreement of the ray and wave methods can, however, be achieved if a sufficient amount of the beam is intercepted by the mirror, as is usually achieved in practice. From our ray and wave simulations, a rough criterion is $r_{m} \gtrsim 3 w$ if there is no mirror tilt or shift. If the mirror is tilted or shifted, the criterion becomes $r_{m} \gtrsim$ $3\left(w+y_{\max }\right)$.

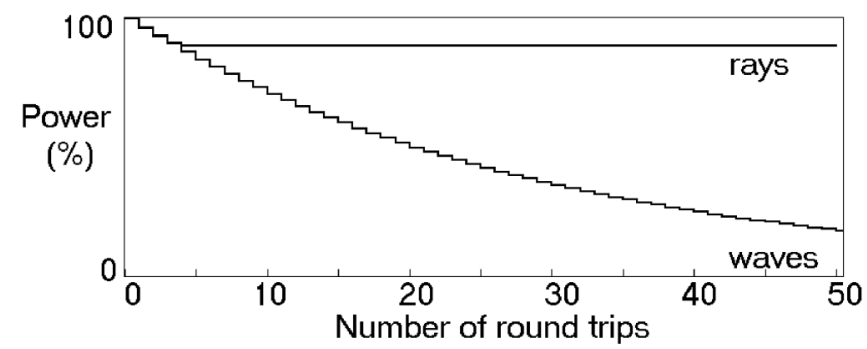

FIG. 8. Power evolution using both ray and wave simulations for 50 round trips of an optical beam in a resonator with finite transverse radius mirrors. For both cases, $z_{0}=0.1$, mirror radius $r_{m}=2$, and $\theta_{m}=h=0$. In the ray simulation, rays not excluded after $\approx 4$ round trips remain in the beam and the power remains constant. In the wave simulation, continued diffraction causes the power to exponentially decrease to zero.

\section{DISCUSSION}

The goal of this paper has been to show how mirror tilt, mirror shift, and focal length change can affect the Gaussian mode of a short Rayleigh length resonator with no gain. All of these effects can be studied using a suitable random collection of rays to emulate the optical beam. In the cases of mirror tilt and transverse shift, the beam tends to rock up and down on the mirrors and, if the rocking amplitude is sufficiently large, to cause the beam position to exceed the transverse mirror radius. In the cases of longitudinal mirror shift and focal length change, the beam will remain on axis but the beam radius at the mirror will expand and contract with successive reflections. Again, if the beam radius becomes too large, portions of the beam may exceed the transverse mirror radius. For mirrors with finite transverse radii, the ray method causes the power to decrease and stabilize at a smaller value; however, wave simulations show that the power decreases uniformly to zero. Agreement between the two methods can be achieved if the transverse mirror radius $r_{m} \gtrsim 3 w$.

In order to better appreciate these results, we apply them to a resonator of length $S=10 \mathrm{~m}$ and $z_{0}=0.1$. From Fig. 3, we find that the maximum transverse shift of the beam on a mirror will be $1 \mathrm{~mm}$ if a mirror tilts by $\theta_{m}=$ $0.1 \mathrm{mrad}$ or shifts transversely by $H=33 \mu \mathrm{m}$. Both distortions are well within current design tolerances. On the other hand, longitudinal mirror shift or focal length change will cause the beam to expand and contract. From Figs. 5 and 7, we find that an expansion of $10 \%$ of the beam width will be caused by longitudinal mirror shift $\Delta S=$ $5 \mathrm{~cm}$ or fractional focal length change $\Delta f / f=1 \%$. Again, these distortions are easily managed by existing technology.

Gain effects are explicitly not covered in this paper; however, gain effects in a short Rayleigh length FEL with mirror misalignment have been reported previously by Crooker, et al. [2] using wave simulations. Results indicate that the electron beam tends to keep the optical beam confined near the electron beam axis, even for significant mirror shift and tilt.

\section{ACKNOWLEDGMENTS}

The authors are grateful for support from NAVSEA, ONR, and the JTO.

[1] W. B. Colson, J. Blau, and R. L. Armstead. Nucl. Instrum. Methods Phys. Res., Sect. A 507, 48 (2003).

[2] P. P. Crooker, T. Campbell, W. Ossenfort, S. Miller, J. Blau, and W. B. Colson. Nucl. Instrum. Methods Phys. Res., Sect. A 507, 52 (2003).

[3] W. B. Colson, in Proceedings of the 25th International FEL Conference, 2004, http://www.jacow.org 
[4] D. Herriott, H. Kogelnik, and R. Kompfner, Appl. Opt. 3, 523 (1964).

[5] A.E. Siegman, Lasers (University Science Books, Sausalito, CA, 1986).

[6] J. B. Rosenzweig, Fundamentals of Beam Physics (Oxford University Press, New York, 2003).

[7] R. Armstead, J. Blau, and W. B. Colson, in Proceedings of the 25th International FEL Conference, 2004, http:// www.jacow.org
[8] G. D. Boyd and J. P. Gordon, Bell Syst. Tech. J. 40, 489 (1961).

[9] J. A. Arnaud, Appl. Opt. 8, 1909 (1969).

[10] A. G. Fox and T. Li, Proc. IEEE 51, 80 (1963).

[11] R. Hauck, H. P. Kortz, and H. Weber, Appl. Opt. 19, 598 (1980).

[12] J. L. Remo, Appl. Opt. 19, 774 (1980).

[13] R. L. Sanderson and W. Streifer, Appl. Opt. 8, 2241 (1969). 